\title{
L'analyse Qualitative Inorganique Dans le Curriculum Formel Marocain et Chez Les Futurs Enseignants de Physique-Chimie en Cycle Secondaire Collégial
}

\author{
Ibrahim Bouabdallah, (Docteur, Enseignant-chercheur)
}

Centre Régional des Métiers de l'Éducation et de la Formation de l'Oriental, Hay Almassira, Oujda, Maroc. Associé au Laboratoire de Chimie Appliquée et Environnement, Faculté des Sciences, Université Mohammed Ier, Oujda,

Maroc

\section{Doi:10.19044/esj.2020.v16n18p188 URL:http://dx.doi.org/10.19044/esj.2020.v16n18p188}

\section{Résumé}

L'analyse qualitative inorganique, une méthode d'analyse chimique qualitative permettant d'identifier les constituants d'une substance sert à illustrer les phénomènes chimiques en cycle secondaire collégial. Les futurs enseignants sont confrontés à des exigences particulières en termes de connaissances relatives à l'objet de savoir, pour pouvoir assurer la transposition didactique. Cet article vise, d'une part, l'examen des contenus à enseigner en chimie aux apprenants du secondaire collégial, et d'autre part, la détermination du niveau d'acquisition des connaissances relatives à l'analyse qualitative inorganique chez les futurs enseignants. Pour ce faire, une analyse exhaustive du curriculum formel marocain, suivie d'un questionnaire ouvert soumis à cinquante-huit futurs enseignants, ont été menés pour collecter les données. Les résultats concernant le curriculum formel montrent l'absence de thèmes consacrés à ce champ disciplinaire qui entretient une corrélation implicite avec les activités d'enseignement. La dissolution et l'identification du gaz formé ne constituent pas un obstacle pour les futurs enseignants. Cependant, ils éprouvent d'énormes difficultés pour expliquer les réactions mises en jeu lors de l'action de la substance inconnue avec les réactifs. En plus, leur savoir-faire pratique révèle une lacune pour proposer un montage expérimental de production du sulfure d'hydrogène.

Mots-clés: Analyse Qualitative Inorganique, Futurs Enseignants, Savoirs Disciplinaires 


\title{
Inorganic Qualitative Analysis in the Moroccan Formal Curriculum and for the Future Physics- Chemistry Teachers in Secondary College Cycle
}

\section{Ibrahim Bouabdallah, (Docteur, Enseignant-chercheur)}

Centre Régional des Métiers de l'Éducation et de la Formation de l'Oriental, Hay Almassira, Oujda, Maroc. Associé au Laboratoire de Chimie Appliquée et Environnement, Faculté des Sciences, Université Mohammed Ier, Oujda, Maroc

\begin{abstract}
The inorganic qualitative analysis, a method of analytical chemistry for identifying the constituents of a substance, is used to illustrate chemical phenomena's in the college secondary cycle. Future teachers are confronted with particular requirements in terms of knowledges related to the knowledge object in order to be able to ensure didactic transposition. This article aims, on one hand, to examine the chemistry contents to be taught in secondary college school, and on the other hand, to determine the future teachers' level of inorganic qualitative analysis knowledge acquisition._To achieve this, an exhaustive analysis of the formal Moroccan curriculum, followed by an openended questionnaire submitted to fifty-eight future teachers, were used to collect the data. The results concerning the formal curriculum show the absence of themes related to this disciplinary field which has correlate with the teaching activities. The dissolution and identification of the formed gas did not appear to be an obstacle for the future teachers. However, they found several difficulties to explain the reactions between the unknown substance and reagents. In addition, their practical knowledge reveals an insufficiency to propose an experimental setup to produce the hydrogen sulfide.
\end{abstract}

Keywords: Inorganic Qualitative Analysis, Future Teachers, Disciplinary Knowledge

\section{Introduction}

Depuis longtemps, l'analyse qualitative inorganique occupe une position centrale dans l'enseignement et l'apprentissage de la chimie. Elle constitue un excellent exercice qui attire la plupart des apprenants (Highton, 1898 : 172). Ainsi, sa pratique durant l'enseignement favorise la formation des habitudes ordonnées, développe le pouvoir d'observation et encourage 
l'utilisation des facultés de raisonnement. Par ailleurs, elle offre de meilleures opportunités à l'enseignant pour faire comprendre à l'apprenant de nombreux principes de la chimie illustrés par le comportement de la matière (Guy, 1928 : 573). En outre, elle contribue au développement des intellectuels en transformant la pensée concrète en une pensée formelle (Shamai \& Stavy, 1986 : 707). Par ailleurs, l'analyse qualitative favorise la formation de l'esprit scientifique chez les apprenants en s'appropriant la démarche scientifique par l'observation, l'enregistrement des effets et la déduction des conclusions (Tan, 2002 : 7). L'analyse qualitative est un outil fondamental pour construire les concepts chimiques et acquérir les compétences pratiques qui construisent un fondement de la recherche scientifique (Theodore, 1929 : 486).

Le recours aux travaux pratiques motive les étudiants et développe le savoir-faire en matière d'investigation et de recherche (Hamlyn et al., 2017 : 35 ). Pour plus d'un siècle, les travaux pratiques ont joué un rôle central dans l'enseignement de la chimie de l'école à l'université (Köller et al., 2015 : 87). Les tâches pratiques ont une signification incontestable dans la perfection de la connaissance et l'acquisition de compétences expérimentales au laboratoire (Parmenov \& Syroezhkin, 1963 : 32). Les apprenants durant les séances pratiques manipulent les produits chimiques, utilisent les appareils et choisissent les techniques et les méthodes appropriées (Tan, $2002: 7$ ).

L'enseignant a un rôle majeur dans l'acquisition de compétences et l'amélioration de la qualité de l'enseignement de la chimie (Bugaje, 2013 : 10). La littérature met en évidence un lien entre les difficultés disciplinaires des enseignants et l'apprentissage lacunaire chez les apprenants (Tan, 2005 : 7 ; Lenton \& Turner, 1999 : 67 ; Valanides, 2000 : 249). Des exigences multiples sont alors imposées aux futurs enseignants de la physique-chimie. Cette étude se propose, d'une part, d'examiner la place qu'accordent les programmes scolaires marocains de l'enseignement de la physique-chimie en cycle secondaire collégial à l'analyse qualitative inorganique, et d'autre part, de déterminer le niveau de compréhension des concepts y afférant chez les futurs enseignants.

\section{Cadre théorique et problématique \\ II.1. Savoir de référence}

L'analyse qualitative est la méthode d'identification des éléments ou des ions qui composent une substance organique ou inorganique sans tenir compte de leurs proportions (Alexéev, 1980 : 11 ; Večeřa \& Gasparič, 1971 : 15 ; Vogel, 1979 : 1; Clyde \& Mary, 1980:1). L'analyse qualitative inorganique nécessite une dissolution pour former des ions représentant la forme la plus simple d'un élément (Syed et al., 2002 : 177). Leur présence dans la solution peut être confirmée lors des réactions avec les réactifs, qui s'accompagnent d'effets externes, facilement reconnaissables, entre autres, le 
changement de couleur, la formation ou la dissolution d'un précipité ou le dégagement d'un gaz (Syed et al., 2002 : 177).

Ces réactifs repartis en deux catégories, possèdent un double rôle (Dessart et al., 1973 : 87 ; Fresenius, $1845: 21$ ). Ils permettent d'une part, l'étude des caractères analytiques des ions, et d'autre part, la détection de la présence des ions en solution. La première catégorie renferme les réactifs généraux utilisés pour classer les ions en groupes. Ainsi, la détermination des classes de cations repose sur les réactifs tels que le chlorure d'hydrogène, le sulfure d'hydrogène, le sulfure d'ammonium et le carbonate d'ammonium, tandis que celle des anions nécessite le nitrate d'argent et le chlorure de baryum. La deuxième catégorie comporte les réactifs spéciaux qui fournissent les caractères particuliers des ions, et permettent l'identification et le contrôle des espèces ioniques présentes en solution, entre autres, l'hydroxyde de sodium, l'iodure de potassium, le dichromate de potassium.

Fresenius (1845:3) rapporte quatre principes pour l'étude de l'analyse qualitative. Le premier stipule l'acquisition d'un savoir faire relatif à l'habitude des opérations chimiques. Par conséquent, l'utilisation des ustensiles qui servent aux manipulations et les produits qui en résultent, constituent des éléments de réussite. Le deuxième principe exige une connaissance suffisante des réactifs et de leur usage. Tandis que le troisième principe insiste sur la matrise de l'action des corps sur les réactifs, permettant ainsi de conclure la nature du corps analysé. Quant au dernier principe, il repose sur la conception de la marche à suivre durant les analyses.

En somme, l'analyse qualitative inorganique nécessite la dissolution de la substance à analyser, suivie de l'action des réactifs selon un processus qui permet la mobilisation des compétences pratiques de manipulation, d'observation et d'interprétation des effets externes.

\section{2. Les difficultés des apprenants et des enseignants}

L'analyse qualitative est un domaine qui pose un défi de taille, aussi bien pour les apprenants en apprentissage, que pour les enseignants chargés de la transposition didactique. Plusieurs difficultés sont rapportées dans la littérature. En effet, Tan et al. (2001 : 223) ont prouvé que les élèves trouvent l'analyse qualitative fastidieuse et difficile à comprendre. En plus, les apprenants déclarent l'absence d'articulations entre les séances pratiques et les apprentissages en classe. Selon eux, l'analyse qualitative exige une grande quantité d'informations à garder dans la mémoire. Outre, ils notent l'existence de difficultés durant les travaux pratiques d'analyse qualitative (Tan et al., 2002 : 69). Aussi, des recherches ont identifié d'autres difficultés rencontrées par les étudiants pour comprendre les concepts liés au sujet des électrolytes, tels que, la solubilité, les réactions ioniques, l'équilibre ionique, l'équilibre acide-base et l'hydrolyse (Johnstone \& Wham, 1982:71; Garforth et al., $1976: 72)$. 
Au sein de la classe, toutes ces difficultés imposent aux acteurs de terrain la mobilisation de plusieurs ressources, en particulier les acquis disciplinaires. Pour les apprenants, la compréhension des réactions qui se produisent lors de l'analyse qualitative nécessite une connaissance tacite des phénomènes et des réactifs impliqués (Tan et al., 2002 : 69). Une autre exigence impose aux apprentis la clarté des procédures, des réactions et des stratégies métacognitives pour expliquer les résultats expérimentaux. Du côté des enseignants, les acquis disciplinaires et les conceptions qu'ils possèdent vis-à-vis des phénomènes enseignés peuvent améliorer ou limiter l'apprentissage des apprenants (Valanides, 2000: 249). Pour cela, les enseignants doivent expliciter les liens entre la théorie que les élèves apprennent en classe et les travaux pratiques effectués au laboratoire (Tan et al., 2002 : 69). Par ailleurs, la réalisation des expériences d'analyse qualitative, leur impose la nécessité de modéliser des stratégies appropriées et des façons de penser, en surveillant bien ce que les apprenants font (Schraw, 1998 : 113). Ainsi, la compréhension des réactions mises en jeu, facilite l'acquisition des concepts, des processus et des capacités de réflexion; ce qui exige peu d'efforts cognitifs, mais rentables en termes de résultats (Tan et al., 2004 : 97).

L'ampleur de ces exigences s'accroit d'avantage pour les futurs enseignants au moment où des études ont montré que ces derniers possèdent des conceptions alternatives similaires à celles des étudiants dans plusieurs domaines (Tan, 2005 : 7), entre autres, l'équilibre chimique (Quilez-Pardo \& Solaz-Portoles, 1995 : 939), les réactions d'oxydo-réduction (De Jong et al., 1995 : 1097) et le comportement des gaz (Lin et al., 2000 : 235). La présence de ces conceptions alternatives chez les futurs enseignants peut nuire à la compréhension conceptuelle et les amener à expliquer le comportement de la matière de façon erronée (Cormier, 2014 : 26). Les futurs enseignants deviennent alors une source de conceptions alternatives de leurs élèves (Tan, 2005 : 7; Wandersee et al., 1994 : 177). Étant donné le nombre d'étudiants formés au cours d'une carrière d'enseignant, la génération de conceptions alternatives peut être assez importante (Valanides, 2000 : 249). Pour pourvoir surmonter les difficultés des apprenants, les acquis disciplinaires chez les futurs enseignants peuvent contribuer à expliquer correctement le comportement de la matière et à faire des prédictions exactes en analyse qualitative inorganique.

\section{II.3. Problématique}

La fonction de l'analyse qualitative inorganique dans l'enseignement et l'apprentissage de la chimie intègre deux composantes : la transposition didactique et la pratique expérimentale. La première composante concerne la pertinence de l'utilisation de l'analyse qualitative dans les cours de chimie où la compréhension des étudiants est renforcée (Cooley \& Williams, 1999 : 
1117). Elle sert de support de base pour illustrer et mettre en évidence les concepts, les corps et les phénomènes chimiques qui se produisent lors des réactions chimiques, entre autres, les réactions d'équilibre, d'acide-base, d'oxydo-réduction et de complexation (Randall \& Holly, 2012 : 254). Les résultats visuels de ces réations, tels que le changement de couleur ou la formation d'un précipité, sont très positifs pour la plupart des étudiants (Cooley \& Williams, 1999 : 1117). La deuxième composante permet au moyen d'expériences simples, claires et faciles de comprendre et maitriser l'action concrète des opérations pratiques (Theodore, 1929: 486). La reconnaissance de son statut avancé dans la construction des concepts, nécessite un examen de la place accordé à l'analyse qualitative dans le curriculum formel de physique-chimie en cycle secondaire collégial (Willame \& Snauwaert, 2015 : 177).

Parallèlement à cela, des exigences particulières d'apprentissage inhérentes à l'objet de savoir sont alors imposées aux futurs enseignants pour pouvoir transformer le contenu à enseigner en un savoir enseigné qui puisse être compris par les élèves (Chevallard, 1985 : 39). La littérature regroupe les connaissances en quatre domaines : pédagogiques, didactiques, curriculaires et disciplinaires (Imène et al., 2011 : 153 ; Nguessan, 2016 : 241). En l'occurrence, l'examen du niveau de compréhension des concepts chez les futurs enseignants constitue un autre support de réussite dans le parcours du savoir du professeur à l'élève.

Ainsi comme nous l'avons précédement cité, l'investigation stipule en premier lieu, analyser les savoirs à enseigner, tels qu'ils apparaissent dans les programmes officiels marocains, et ensuite, avancer dans la détermination du niveau de compréhension des connaissances disciplinaires nécessaires aux futurs enseignants de physique-chimie en analyse qualitative inorganique. Les indicateurs examinés dans ce travail se réfèrent, au début, à l'effet de la forme métallique sur la dissolution en milieu acide et l'identification du gaz formé ; ensuite l'explication des réactions mises en jeu lors de l'action de la solution avec le nitrate d'argent, le sulfure d'hydrogène, l'hydroxyde de sodium ; et enfin le savoir faire expérimental pour produire un montage de manipulation de $\mathrm{H}_{2} \mathrm{~S}$.

Les résultats issus de l'investigation permettent la sensibilisation des futurs enseignants à l'importance accordée à l'analyse qualitative inorganique dans l'enseignement de la chimie. Ce qui aide à mettre en œuvre des stratégies capables d'améliorer la formation des enseignants.

Notre problématique oscille autour de la place accordée à l'analyse qualitative inorganique dans le curriculum formel marocain de physiquechimie en cycle secondaire collégial et l'examen du niveau de compréhension des savoirs disciplinaires chez les futurs enseignants. 
Pour répondre à cette problématique, une analyse des programmes scolaires pour déterminer les savoirs à enseigner et les relations qu'ils entretiennent avec les principes de l'analyse qualitative inorganique, a été effectuée dans un premier temps. Ensuite, la caractérisation du niveau de compréhension des savoirs savants chez les futurs enseignants de physiquechimie en cycle secondaire, a été entamée via un questionnaire ouvert.

\section{Méthodologie et procédure}

\section{III.1. Méthodologie}

Cette étude fait suite à un projet de recherche plus vaste qui stipule une évaluation des performances des cadres d'enseignement en cycle secondaire. La méthodologie adoptée a été basée sur une analyse exhaustive du curriculum formel marocain pour l'enseignement de la physique-chimie en cycle secondaire collégial et un questionnaire élaboré à partir des éléments identifiés dans le cadre théorique.

\section{III.2. Population et échantillon}

La population visée est constituée de futurs enseignants de physiquechimie en cycle secondaire, ayant accompli durant l'année 2018-2019, leur qualification professionnelle pour exercer le métier d'enseignant dans des établissements d'enseignement secondaire collégial ou qualifiant. L'échantillon choisi est composé d'une cohorte de cinquante-huit (58) futurs enseignants ( 35 hommes et 23 femmes) de physique-chimie qui sont admis au Centre Régional des Métiers de l'Éducation et de la Formation de l'Oriental pour prendre en charge les apprenants aux seins des établissements scolaires l'année suivante. Les participants ont suivi 68 heures de compléments formation en chimie durant leur qualification. Ils sont âgés entre 22 et 45 ans et titulaires d'une licence (13 en chimie, 44 en physique, 1 en didactique de physique-chimie).

\section{III.3. Description et administration du questionnaire}

Le questionnaire construit sur la base de 4 tests (Tableau 1), comporte des questions ouvertes qui s'articulent autour de cinq thèmes en liens avec la dissolution des métaux (fer, cuivre, zinc, aluminium) programmés en cycle secondaire collégial (MENFP, 2015) (Q1); l'analyse d'un gaz (Q2) ; l'identification d'un anion (Q3-4) et d'un cation (Q5-7); et la conduite expérimentale d'une réaction de contrôle (Q8-9) (Cetin-Dindar \& Geban, 2011 : 600 ; Calik \& Ayas, 2005 : 638 ; Chou, 2002 : 73). 
Tableau 1 : Tests, observations et questions

\begin{tabular}{|c|c|c|c|}
\hline Étapes & Tests & Observations & Questions \\
\hline A & $\begin{array}{l}\text { On verse un excès d'acide } \\
\text { chlorhydrique concentré sur } \\
0,5 \mathrm{~g} \text { d'un métal gris en } \\
\text { feuilles. Après dissolution } \\
\text { totale et dilution, le contenu } \\
\text { est ré- parti en trois tubes à } \\
\text { essais A, B et C. }\end{array}$ & $\begin{array}{l}\text { Dégagement gazeux } \\
\text { incolore qui brûle à } \\
\text { l'approche d'une } \\
\text { allumette enflammée. } \\
\text { La réaction devient } \\
\text { violente et la solution } \\
\text { prend une teinte grise, } \\
\text { puis incolore. }\end{array}$ & $\begin{array}{l}\text { Q1. Expliquer le choix en } \\
\text { feuilles par rapport aux autres } \\
\text { formes? } \\
\text { Q2. Identifier le gaz formé en } \\
\text { (a)? }\end{array}$ \\
\hline B & $\begin{array}{l}\text { Le contenu de A est traité } \\
\text { par quelques gouttes d'une } \\
\text { solution de nitrate d'argent. }\end{array}$ & $\begin{array}{l}\text { Formation d'un } \\
\text { précipité blanc qui } \\
\text { noircit à la lumière. }\end{array}$ & $\begin{array}{l}\text { Q3. Interpréter la formation } \\
\text { du précipité formé en (b) ? } \\
\text { Q4. Interpréter l'effet de la } \\
\text { lumière sur le précipité ? }\end{array}$ \\
\hline $\mathrm{C}$ & $\begin{array}{l}\text { Le contenu de B est traité } \\
\text { par une solution } \\
\text { d'hydroxyde de sodium. }\end{array}$ & $\begin{array}{l}\text { Obtention d'un précipité } \\
\text { blanc qui se transforme } \\
\text { sous la chaleur en } \\
\text { monoxyde de métal. }\end{array}$ & $\begin{array}{l}\text { Q5. Identifier le métal? } \\
\text { Q6. Écrire l'équation de } \\
\text { précipitation? } \\
\text { Q7. Donner l'équation } \\
\text { traduisant la formation du } \\
\text { monoxyde de métal? }\end{array}$ \\
\hline $\mathrm{D}$ & $\begin{array}{l}\text { On ajoute à } \mathrm{C} \text { un mélange } \\
\text { de } \mathrm{NH}_{4} \mathrm{Cl} / \mathrm{NH}_{3} \text { et on barbote } \\
\text { le sulfure d'hydrogène. }\end{array}$ & $\begin{array}{l}\text { Apparition d'un } \\
\text { précipité blanc. }\end{array}$ & $\begin{array}{l}\text { Q8. Proposer un montage } \\
\text { expérimental pour barboter } \\
\mathrm{H}_{2} \mathrm{~S} \text { ? } \\
\text { Q9. Identifier le précipité ? }\end{array}$ \\
\hline
\end{tabular}

Après la validation par expérimentation, le questionnaire a été soumis de façon anonyme en fin d'année de qualification professionnelle, en présence du chercheur, aux futurs enseignants qui ont pris entre 30 et 45 minutes pour répondre.

\section{Résultats et discussion \\ IV.1. L'analyse des programmes scolaires}

Le curriculum formel marocain ne consacre aucun thème du programme de physique-chimie à l'analyse qualitative inorganique en cycle secondaire collégial (MENFP, 2015). Cependant, les domaines choisis constituent une base importante dans la construction de concepts chimiques. L'importance accordée aux savoirs et savoir-faire en lien avec l'analyse qualitative, est marquée par le choix des dirigeants éducatifs pour améliorer les compétences des apprenants, en particulier l'acquisition des concepts et la conception des démarches efficaces pour résoudre les problèmes en chimie. 
En effet, le concept de dissolution est examiné en première année. Il fait référence au mélange homogène qui peut être obtenu à partir des solutés à l'état solide, liquide ou gaz. En plus, les savoirs expérimentaux sont acquis durant toutes les activités pratiques. Par ailleurs, les autres indicateurs sont concentrés au niveau de la troisième année du cycle d'enseignement secondaire collégial. Les orientations officielles les présentent via deux dimensions dont l'horaire légal accordé aux activités d'enseignement est fixé à 6 heures. Ce qui représente environ $30 \%$ de l'horaire total du programme de chimie.

La première dimension porte sur l'étude des réactions chimiques des matériaux sur les solutions. Selon cette vision, les réactions chimiques de quelques métaux typiques avec des solutions acides et des solutions basiques sont présentées aux apprenants en utilisant des activités expérimentales. Pour se faire, les actions de la solution concentrée de l'acide chlorhydrique sur le fer, le cuivre, le zinc et l'aluminium sont étudiées. Ce qui permet aux apprenants de dégager les caractéristiques fondamentales de ces réactions, en particulier le phénomène de dissolution des composés métalliques et la caractérisation des produits formés. Ces derniers sont identifiés par les apprenants, ce qui induit à l'écriture des équations bilans des réactions sous la forme la plus simple en respectant les règles de la symbolisation et de la conservation de la matière, par exemple : $\mathrm{Fe}+2 \mathrm{H}^{+} \rightarrow \mathrm{Fe}^{2+}+\mathrm{H}_{2}$. De façon similaire, les réactions des mêmes métaux avec une solution d'hydroxyde de sodium sont effectuées. En accord avec cette situation, les seuls métaux qui réagissent avec la solution basique sont le zinc et l'aluminium. Le dihydrogène formé est mis en évidence par les apprenants au moyen de l'action d'une étincelle qui produit une petite détonation.

La deuxième dimension porte sur l'identification expérimentale des ions en solution au moyen de tests spécifiques basés sur les réactifs spéciaux. Ainsi, l'ion $\mathrm{Cu}^{2+}$ appartenant au groupe II et les cations $\mathrm{Zn}^{2+}, \mathrm{Al}^{3+}, \mathrm{Fe}^{2+}$, $\mathrm{Fe}^{3+} \mathrm{du}$ groupe III sont caractérisés par l'action d'une solution d'hydroxyde de sodium. Tandis que l'identification de l'anion $\mathrm{Cl}^{-}$appartenant au groupe I est réalisée au moyen du nitrate d'argent. Durant ces activités pratiques, les apprenants élaborent les équations bilans de précipitation. En parallèle, le même référentiel insiste sur la nécessité de motivation des élèves pour réaliser d'autres tests d'identification des ions en solution selon un protocole expérimental bien défini. Ces activités proposées par les élèves, permettent l'acquisition des compétences pratiques, en particulier l'inventaire des matériels nécessaires pour réaliser les manipulations. 
$\mathrm{Au} \mathrm{Maroc,} \mathrm{aucun} \mathrm{thème} \mathrm{concernant} \mathrm{l'analyse} \mathrm{qualitative} \mathrm{inorganique}$ n'est inclus de façon explicite dans le curriculum formel de physique-chimie en cycle secondaire collégial. Pourtant, les activités pratiques réelles y afférentes, en particulier la dissolution des métaux (fer, cuivre, zinc, aluminium) via l'action des acides et des bases, l'identification des cations et de l'anion chlorure, y occupent une place importante pour construire les concepts chimiques de base. D'où la nécessité de maitrise du corps d'enseignement des connaissances disciplinaires suffisantes pour assurer efficacement la transposition didactique des savoirs savants.

\section{IV.2. Le savoir savant des futurs enseignants}

Les résultats collectés sur le Tableau 2 sont classés en quatre types de réponses : les correctes $(\mathrm{C})$; les fausses $(\mathrm{F})$; les partielles $(\mathrm{P})$; et les nonréponses $(\mathrm{NR})$.

Tableau 2: Fréquences des réponses par rapport à l'échantillon

\begin{tabular}{cccccccccc}
\hline Catégories & $Q 1$ & $Q 2$ & $Q 3$ & $Q 4$ & $Q 5$ & $Q 6$ & $Q 7$ & $Q 8$ & $Q 9$ \\
\hline$C$ & 36 & 51 & 6 & 1 & 8 & 5 & 2 & 2 & 2 \\
$F$ & 14 & 7 & 20 & 7 & 34 & 44 & 12 & 7 & 7 \\
$P$ & 4 & 0 & 27 & 7 & 0 & 0 & 0 & 5 & 5 \\
$N R$ & 4 & 0 & 5 & 43 & 16 & 9 & 44 & 44 & 44 \\
\hline Total & 58 & 58 & 58 & 58 & 58 & 58 & 58 & 58 & 58 \\
\hline
\end{tabular}

Les réponses montrent que $62,1 \%$ et $87,9 \%$ des futurs enseignants ont répondu correctement aux questions $\mathrm{Q} 1$ et $\mathrm{Q} 2$ respectivement, indiquant une certaine familiarité avec les concepts induits par ces items, tandis que la fréquence de réponses correctes est très faible pour le reste des questions avec des différences statistiquement significatives et des similitudes très nettes pour Q4 et Q7-9. Le taux de réponses correctes varie entre la valeur la plus faible obtenue pour Q4 (1/58) et la plus haute enregistrée pour Q5 (8/58). En plus, le taux observé de non-réponses reste très élevé pour les questions Q4 et Q7-9 mettant en évidence la présence d'une carence chez les questionnés. L'abstention explicite permet probablement au futur enseignant de noter qu'il ne connaît pas la réponse. Une analyse plus fine des scores obtenus révèle l'existence de difficultés majeures pour interpréter les résultats expérimentaux obtenus par l'action du nitrate d'argent, du sulfure d'hydrogène et de l'hydroxyde de sodium sur la substance étudiée. Les analyses des réponses aux différentes questions et la discussion seront regroupées sous les thèmes suivants : l'influence de la forme métallique sur la dissolution, l'identification d'un gaz, d'un anion, d'un cation et la conduite expérimentale d'une réaction de contrôle. 


\section{IV.2.1. Influence de la forme métallique sur la dissolution}

La question relative à la mise en solution partielle ou totale de l'échantillon soumis à l'étude constitue une étape cruciale dans l'analyse qualitative inorganique (Vogel, 1979 : 405 ; Mendham et al. 2006 : 111). Elle permet aux apprenants de découvrir et de connaitre l'action de l'acide chlorhydrique sur le métal (MENFP, 2015). À partir du principe selon lequel, la réactivité dépend de la surface de contact entre les réactifs, la littérature rapporte l'existence d'une relation proportionnelle entre la vitesse d'attaque d'un réactif à l'état solide et la surface du corps qui augmente avec les opérations de division (Charlot, 1974: 317). Ainsi, la fragmentation d'un solide en particules plus fines, entraine une augmentation de la surface de contact qui accroit le nombre de collisions, ce qui conduit à une élévation de la vitesse de réaction.

Les métaux que les futurs enseignants manipuleront avec leurs élèves aux seins des laboratoires scolaires se présentent sous plusieurs formes, entre autres, la limaille, les tournures, les feuilles, les poudres, les fils, les laines et les grains. L'utilisation de l'une de ces variétés devrait leur permettre de mobiliser les connaissances disciplinaires pour justifier leurs choix, en particulier, le contrôle et les propriétés cinétiques de ces réactions exploitées en enseignement de la chimie, ainsi que les mesures de sécurité à prendre. En se servant de ce cadre de travail, l'analyse des réponses montre que les conceptions de plus de la moitie des enseignants (36/58) résonnent avec les principes ci-dessus. Cependant, un quart des futurs enseignants répond de façon erronée et relève des lacunes disciplinaires très graves (14/58). Plusieurs erreurs conceptuelles sont fréquemment rencontrées : la forme du métal inhibe la réactivité : «le métal en feuilles ne réagit pas avec l'acide chlorhydrique 》; la confusion entre la mise en solution du métal par une attaque acide dans un milieu humide et la réaction de combustion qui nécessite un apport d'oxygène : «les feuilles aident à la combustion de façon rapide »; l'absence chez le futur enseignant de la réalité concrète de l'expérience proposée : «les autre formes permettent la dispersion de l'acide chlorhydrique »; la difficulté à identifier l'objectif de l'expérience : "on ne peut pas utiliser la poudre car on risque la dissolution du métal dans la solution »; l'utilité du calcul de la surface des feuilles dans une réaction d'oxydation : "déterminer la surface de la feuille ».

\section{IV.2.2. Identification d'un gaz}

Au moyen de tests simples, plusieurs ions peuvent être caractérisés par l'identification des composés gazeux les plus utilisés dans les laboratoires scolaires, entre autres, le dioxygène, le dioxyde de carbone, le dihydrogène, le dichlore et le sulfure d'hydrogène (Vogel, 1979 : 168). La question relative à l'identification d'un gaz est la plus bien réussie par les futurs enseignants 
avec un taux de non-réponse nul. Elle implique la maitrise des techniques de caractérisation des gaz usuels que les apprenants sont motivées de connaitre. La majorité des questionnés (51/58) réponde correctement, en affirmant la formation de $\mathrm{H}_{2}$. Certains ont même explicité la demi-équation de réduction de l'ion hydrogène $\left(2 \mathrm{H}^{+}+2 \mathrm{e}^{-} \rightarrow \mathrm{H}_{2}\right)$. Ainsi, le brûlage du gaz formé, accompagné d'une légère explosion, caractéristique du dihydrogène, semble être une image bien imprégnée dans la tête des futurs enseignants.

Cependant, l'analyse des réponses fausses (7/58) permet de tirer plus d'informations sur les savoirs disciplinaires chez le futur enseignant. L'une révèle une confusion pour prévoir la catégorie organique ou minérale des espèces chimiques issues de l'action d'un acide sur un métal : "l'action de l'acide chlorhydrique sur les feuilles métalliques conduit à la formation du méthane ». D'autres réponses ont stipulé la formation des gaz $\mathrm{O}_{2}$ et $\mathrm{CO}_{2}$, ce qui illustre l'incapacité à exploiter la loi qualitative de conservation de la matière pour identifier le gaz formé. Pour deux futurs enseignants qui ont prévu la formation du $\mathrm{Cl}_{2}$, la distinction entre les ions actifs et les ions spectateurs constitue un autre obstacle. Il convient enfin de noter que la difficulté à déterminer les espèces chimiques engagées dans la réaction limite la capacité des interrogés à interpréter le dégagement gazeux produit lors de la réaction du métal avec les réactifs.

\section{IV.2.3. Identification d'un anion}

Cette partie très instructive au niveau du phénomène de la précipitation (Nakiboğlu \& Nakiboğlu, 2019 : 873) intègre l'action d'un réactif de groupe (nitrate d'argent) sur l'anion chlorure présent en solution (Q3), suivie de l'effet de la lumière solaire sur le précipité blanc (Q4).

Contrairement aux résultats obtenus lors des questions Q1 et Q2, la situation pour le premier volet de cette partie devient plus compliquée pour les futurs enseignants. En effet, le nombre de réponses fausses et partielles est plus important, et le taux de réponses correctes est plus faible. Seule une minorité des répondants (6/58) a réussi l'interprétation de la formation du précipité blanc correspondant au chlorure d'argent qui confirme la présence de l'anion $\mathrm{Cl}^{-}$appartenant au groupe I. Cette acquisition a été traduite au niveau symbolique (Johnstone, 1993 : 701), par l'équation bilan explicitant la réaction de précipitation $\left(\mathrm{Ag}^{+}+\mathrm{Cl}^{-} \rightarrow \mathrm{AgCl}\right)$. Cependant, l'analyse des réponses fausses d'un tiers $(20 / 58)$ des futurs enseignants traduit l'existence de plusieurs conceptions erronées: l'incapacité à mobiliser les acquis antérieurs relatifs aux propriétés chimiques des acides inorganiques, en particulier l'état physique : «l'acide chlorhydrique précipite »; la confusion entre l'élément chimique et l'ion correspondant, illustre la difficulté d'identifier l'état physique du dichlore: «le précipité blanc indique la présence du chlore (ion chlorure) »; l'incapacité à exploiter l'action du réactif 
du premier groupe des anions pour prévoir les réactions probables : « lorsqu'on ajoute la solution du nitrate d'argent au contenu du tube à essais, il réagit l'ion d'argent avec les ions de l'aluminium pour former le précipité blanc d'oxyde d'aluminium $\mathrm{AlO}_{3} \gg$; les difficultés à pronostiquer les ions issus de la dissolution du nitrate d'argent et les valences des ions usuels : «la formation de l'ion $\mathrm{Ag}^{3+}$ »; la difficulté à mobiliser les nombres d'oxydation et à interpréter la précipitation d'un composé : « $\left(\mathrm{Zn}^{2+}, 2 \mathrm{Cl}^{-}\right)+\left(\mathrm{Ag}^{+}, \mathrm{NO}_{3}^{-}\right) \rightarrow$ $\mathrm{AgCl}_{2}+\mathrm{ZnNO}_{3} »$; une mauvaise assimilation du concept de précipité «le précipité blanc est le $\mathrm{Cl}^{-} \gg$. Concernant les réponses incomplètes qui ont réalisé le meilleur score (27/58), les questionnés ont réécrit le mode opératoire du questionnaire sans présenter aucun élément de réponse. Toutes ces difficultés qui coexistent dans l'esprit des futurs enseignants rendent la tâche d'interprétation des comportements chimiques des matériaux très délicate.

Pour le deuxième volet de cette partie, les tendances s'aggravent davantage quand les futurs enseignants s'expriment sur l'identité des produits formés lors de l'évolution dans le temps du chlorure d'argent. En effet, le nombre de réponses correctes est très faible. Un seul futur enseignant répond correctement en affirmant la transformation du chlorure d'argent, via une réaction d'oxydo-réduction, en argent métallique et en dichlore $(\mathrm{AgCl} \rightarrow \mathrm{Ag}$ $+\mathrm{Cl}_{2}$ ), tandis que les réponses fausses et les réponses partielles ayant réalisées des scores identiques (7/58), ont subi des diminutions très nettes par rapport à ceux observées pour la question précédente. En outre, 74,1\% des futurs enseignants de l'échantillon n'ont ni commenté, ni interprété le noircissement du produit issu de la précipitation, et se sentent donc incapables de fournir des informations pertinentes. Par ailleurs, les erreurs relevées parmi les réponses fausses ou partielles montrent la persistance de lacunes conceptuelles. En plus, la formation et la qualification n'ont pas permis à ces futurs enseignants de construire une représentation satisfaisante de cette réaction caractéristique du chlorure d'argent. Un enseignant a assimilé le corps noir au carbone sans faire recours au bilan qualitatif des réactifs mis en jeu : "la formation du précipité blanc après l'ajout du nitrate d'argent prouve la présence du carbone ». Un autre n'a pas pu comprendre les résultats expérimentaux : "l'ion $\mathrm{Cl}^{-}$devient noir sous l'effet de la lumière ». Un questionné a expliqué le noircissement par la formation des ions qui n'existent pas : «formation des ions $\mathrm{Ag}^{3+}$ ». Enfin, deux autres futurs enseignants ont lié le noircissement du précipité blanc à la formation de l'argent métallique, mais ils n'ont pas pu identifier l'agent réducteur : «formation de l'argent». Ce résultat souligne encore une fois la difficulté à prévoir le transfert électronique entre deux couples d'oxydoréduction. 


\section{IV.2.4. Identification d'un cation}

L'exploitation des résultats obtenus pour l'etape (c) nécessite l'analyse de deux observations. L'une est liée à la précipitation du cation métallique. L'autre décrit l'effet de la chaleur qui transforme le précipité en monoxyde de métal. Cette partie est organisée en trois questions. La première vise à identifier le métal analysé en exploitant les résultats obtenus (Q5) et les deux dernières font références aux équations bilans de précipitation (Q6) et de déshydrations (Q7).

En ce qui concerne la première composante, si trente quatre $(58,6 \%)$ futurs enseignants ont fourni des réponses fausses, à peine huit $(13,8 \%)$ des questionnés répondent correctement en citant le zinc comme étant le métal analysé, contre seize $(27,6 \%)$ futurs enseignants qui ont choisi de ne pas faire de commentaire. Parmi les réponses fausses, on relève quelques erreurs à propos du concept de "métal". Deux futurs enseignants considèrent le soufre, appartenant à la famille des chalcogènes, comme un élément métallique, "le métal analysé le soufre». Encore plus, des chevauchements entre les termes "métal", "oxyde de métal" et "hydroxyde de métal" sont identifiés, «le métal analysé est $\mathrm{Al}(\mathrm{OH})_{3} ; \mathrm{AlO} ; \mathrm{ZnO} ; \mathrm{Al}_{2} ; \mathrm{AlOH} »$. En outre, deux autres réponses illustrent l'incapacité des questionnés à différencier entre la forme oxydée et la forme réduite d'un composé métallique, "le métal mis en solution est l'oxyde de zinc ou l'oxyde d'aluminium». Finalement, certains futurs enseignants ont des difficultés sur le plan symbolique, "aluminium: $\mathrm{Ag}$; $\mathrm{AlO} ; \mathrm{Al}(\mathrm{OH})_{2} ; \mathrm{AlOH} ; \mathrm{Al}_{2} ; \mathrm{Al}^{2+} ; \mathrm{S}^{2+}{ }$.

À propos de la réaction de précipitation des ions zinciques avec l'hydroxyde de sodium (Q6), une minorité des futurs enseignants $(5 / 58)$ a réussi l'écriture de l'équation-bilan $\left(\mathrm{Zn}^{2+}+2 \mathrm{HO}^{-} \rightarrow \mathrm{Zn}(\mathrm{OH})_{2}\right)$. Cependant, la fréquence des réponses incorrectes a augmenté de façon inattendue (44/58). Nous nous limitons à fournir quelques exemples d'erreurs relevées lors de la correction des productions : un futur enseignant a confondu entre l'analyse des tests effectués en (b) et (c), "l'équation-bilan de la réaction est $\mathrm{Ag}^{+}+\mathrm{Cl}^{-} \rightarrow$ $\mathrm{AgCl} \gg$; un autre futur enseignant n'a pas respecté la règle de l'octet pour l'atome du soufre $(Z=16)$ qui cherche à s'entourer de 8 électrons de valence, "l'équation-bilan de la réaction est $\mathrm{S}^{2+}+2 \mathrm{HO}^{-} \rightarrow \mathrm{S}(\mathrm{OH})_{2}$ »; un questionné a considéré que le métal se présente en solution sous la forme réduite, sans tenir compte des règles appliquées pour équilibrer les équations chimiques, «l'équation-bilan est : $\mathrm{Al}+3 \mathrm{HO}^{-} \rightarrow \mathrm{O}_{2}+\mathrm{Al}^{2+}+\mathrm{H}_{3} \mathrm{O}^{+}$»; un autre futur enseignant n'a pas pu identifier la situation de départ, "l'équation-bilan est : $2\left(\mathrm{H}^{+}, \mathrm{Cl}^{-}\right)+\mathrm{S}_{(s)}+\mathrm{H}_{2} \mathrm{O} \rightarrow \mathrm{SO}_{(s)}+2 \mathrm{H}_{2(g)}$ »; d'autres futurs enseignants ont analysé incorrectement les données en estimant la formation des hydroxydes divers tels que, « $\mathrm{Al}(\mathrm{OH})_{3}, \mathrm{AlOH}, \mathrm{Fe}(\mathrm{OH})_{2}, \mathrm{AgOH}, \mathrm{Cu}(\mathrm{OH})_{2} »$.

$\mathrm{Au}$ sujet de l'effet de la chaleur (Q7), qui déshydrate l'hydroxyde de zinc et conduit à la formation du monoxyde de zinc $\left(\mathrm{Zn}(\mathrm{OH})_{2} \rightarrow \mathrm{ZnO}+\mathrm{H}_{2} \mathrm{O}\right)$, 
les futurs enseignants réalisent des résultats insatisfaisants. Deux futurs enseignants ont répondu correctement, tandis que la majorité des questionnés (44/58) ne répond pas à la question. Parmi les données erronées collectées à partir des réponses fausses (12/58), nous relevons les erreurs suivantes : la déshydratation de composés inconvenables, « $\mathrm{Fe}(\mathrm{OH})_{2} \rightarrow \mathrm{FeO}+2 \mathrm{H}_{2} \mathrm{O}$; $\mathrm{S}(\mathrm{OH})_{2} \rightarrow \mathrm{SO}+\mathrm{H}_{2} \mathrm{O}$ »; la non maitrise de la réaction de déshydratation de l'hydroxyde de zinc, "sous l'effet de la chaleur on transforme le mélange $\left(\mathrm{O}_{2}\right.$ $\left.+\mathrm{Al}^{2+}+\mathrm{H}_{3} \mathrm{O}^{+}\right)$en $\mathrm{AlO} ; \mathrm{Al}(\mathrm{OH})_{2} \rightarrow \mathrm{AlO}+\mathrm{H}_{2} ; \mathrm{Zn}+2 \mathrm{H}^{+} \rightarrow \mathrm{Zn}^{2+}+\mathrm{H}_{2}$; $\mathrm{AlOH} \rightarrow \mathrm{AlOH} ; \mathrm{AgCl} \rightarrow \mathrm{AgO} » ;$ la confusion entre les réactions de précipitation et de déshydratation, « $\left(\mathrm{Zn}^{2+}, 2 \mathrm{Cl}^{-}\right)+\mathrm{OH}^{-} \rightarrow \mathrm{ZnO}+2 \mathrm{Cl}^{-}+\mathrm{H}^{+}$; $2 \mathrm{HO}^{-}+2 \mathrm{Al}^{+} \rightarrow 2 \mathrm{AlO}+\mathrm{H}_{2} \mathrm{O} ; \mathrm{HCl}+\mathrm{Fe}+\mathrm{NaOH} \rightarrow \mathrm{H}_{2}+\mathrm{FeO}+\mathrm{NaCl} »$.

\section{IV.2.5. La conduite expérimentale d'une réaction de contrôle}

Dans le dernier test (d), nous avons demandé aux futurs enseignants de proposer un montage expérimental pour manipuler un réactif de groupe en vue d'une réaction de contrôle. Notre choix s'est fixé sur le barbotage du sulfure d'hydrogène dont la connaissance des propriétés, implique une utilisation raisonnée en matière d'impact environnemental (Q8). Ensuite, le questionné s'assure de l'existence de l'ion $\mathrm{Zn}^{2+}$ par l'identification de la nature du précipité (Q9).

Les résultats observés pour le premier axe montrent que seulement deux futurs enseignants ont proposé les éléments nécessaires pour faire réagir ce réactif du groupe avec l'ion zincique, tandis que les trois-quarts (44/58) de la population n'ont pas tenté de produire un montage expérimental correct. Les futurs enseignants ne disposent pas d'acquis nécessaires au niveau de l'utilisation des principaux matériels didactiques expérimentaux, ce qui démontre la faible percée de ce savoir pourtant essentiel dans l'analyse qualitative inorganique. Parmi les réponses erronées, nous identifions quelques difficultés. Tout d'abord, la proposition de montages expérimentaux qui ne tiennent pas compte de l'état gazeux du sulfure d'hydrogène. En plus, ils ne respectent pas les normes de sécurité relatives à ce réactif (Vogel, 1979 : 166). Les futurs enseignants s'avèrent inconscients du caractère polluant du sulfure d'hydrogène. Ensuite, les réponses montrent explicitement une incohérence entre le matériel proposé et ses utilisations les plus fréquentes. Ainsi, un futur enseignant a choisi de mettre la solution $\mathrm{C}$ dans un cristallisoir pour barboter $\mathrm{H}_{2} \mathrm{~S}$. Un autre questionné propose de mettre le gaz réactif dans une fiole. Enfin, les futurs enseignants se montrent incapables de présenter des montages expérimentaux au moyen de schémas qui facilitent la construction des connaissances (Givry \& Andreucci, 2015 : 119). Ils ne respectent pas les principes fondamentaux, en particulier, la propreté, la lisibilité, les conventions, la légende, les règles schématisation. Donc, le faible degré de familiarité avec le matériel du laboratoire pourrait avoir des répercussions 
négatives sur les activités expérimentales considérées comme essentielles dans l'acte d'enseignement et constituées un principe majeur dans l'analyse qualitative.

Durant la réaction de contrôle du cation en solution, les questionnés identifient la nature du précipité via un langage écrit ou symbolique. Pour cette composante, les futurs enseignants se sont peu exprimés et des résultats insatisfaisants sont observés. En effet, la majorité des futurs enseignants (44/58) n'ont pas pu interpréter ce résultat expérimental et, par suite, n'ont pas répondu à la question. Seule deux répondants (2/58) ont évoqué la précipitation du sulfure de zinc $(\mathrm{ZnS})$. En plus, le nombre de réponses incorrectes est relativement élevé (7/58). Au terme de cette performance non encourageante pour prévoir le solide qui précipite lors du mélange des deux réactifs antagonistes, il est possible d'affirmer que les difficultés des futurs enseignants tendent à diverger. Un futur enseignant a estimé la possibilité de précipitation des gaz dissous : «le précipité est de nature gazeuse ; le précipité formé est $N_{2}$ ». Un autre considère que le sulfure d'hydrogène, selon ces conditions, réduit l'ion $\mathrm{Zn}^{2+}$ : «le précipité formé est le solide $\mathrm{Zn}$ ». Plus grave aussi, le gaz injecté dans la solution diminue la solubilité, ce qui conduit à la précipitation d'un chlorure de métal: « $\mathrm{Fe}^{2+}+2 \mathrm{Cl}^{-} \rightarrow \mathrm{FeCl}_{2(s)} 》$. Encore plus, des répondants ont expliqué la formation du précipité par des produits non conforme : « $\mathrm{NH}_{4} \mathrm{Cl}$; mélange Fer/Soufre; $\mathrm{FeS} ; \mathrm{AgCl} ; \mathrm{AgSH}_{3(s)}$ ». Enfin, cette question a fait ressortir les problèmes que posent la symbolisation pour les questionnés : « $\mathrm{SH}_{4}+\mathrm{Ag}+\rightarrow \mathrm{AgSH}_{3(\mathrm{~s})}+\mathrm{H}^{+} ; 7 \mathrm{~S}+2 \mathrm{NH}_{3}+\left(\mathrm{H}^{+}, \mathrm{Cl}^{-}\right.$ )$\rightarrow 7 \mathrm{SH}+\mathrm{N}_{2}$ ». En définitive, les futurs enseignants n'arrivent pas à élaborer l'équation bilan de la réaction entre un réactif de groupe et le cation du zinc $\left(\mathrm{Zn}^{2+}+\mathrm{S}^{2-} \rightarrow \mathrm{ZnS}\right)$. Les résultats obtenus, justifient les difficultés des futurs enseignants pour produire une équation-bilan considérée comme un concept intégrateur source de difficultés persistantes chez les apprenants (Barlet \& Plouin, 1994 : 27).

\section{Conclusion}

Durant l'enseignement de la chimie, les futurs enseignants sont amenés à utiliser la démarche expérimentale pour expliciter les concepts chimiques. L'analyse qualitative inorganique leur permet d'inculquer à l'apprenant de nombreux principes fondamentaux, illustrés par l'examen du comportement des métaux. En accord avec cette stratégie, l'utilisation de l'analyse qualitative inorganique n'est pas simple puisqu'il demande un savoir disciplinaire suffisant pour pouvoir accéder à la compréhension des propriétés de la matière. Cet article met en évidence quelques aspects liés à l'enseignement et l'apprentissage de la chimie. Il s'agit d'examiner, au début, le statut de l'analyse qualitative inorganique dans le curriculum formel marocain de physique-chimie en cycle secondaire collégial, ensuite déterminer le niveau 
d'acquisition des concepts chez les futurs enseignants au moyen d'un questionnaire composé des items qui visent : la corrélation entre la forme métallique et la dissolution, l'analyse d'un effet externe, l'identification d'un anion et d'un cation, et la conduite expérimentale d'une réaction de contrôle.

Les réponses collectées pour le premier axe révèlent, que le curriculum formel marocain ne comprend aucun thème concernant l'analyse qualitative inorganique. Cependant, la construction des concepts chimiques de base fait référence à plusieurs activités pratiques y afférentes, telles que la dissolution des métaux (fer, cuivre, zinc, aluminium) dans les acides et les bases, l'identification des cations et des anions. D'où le nouveau corps d'enseignement, doit posséder les connaissances disciplinaires suffisantes en analyse qualitative inorganique pour pouvoir assurer efficacement la transposition didactique des savoirs savants.

Les résultats obtenus pour le deuxième axe, révèlent que les futurs enseignants disposent d'un niveau de compréhension élevé vis-à-vis de l'influence de la forme métallique sur la dissolution dans les acides et l'analyse des gaz issus de cette réaction. Cependant, des conceptions erronées pouvant avoir un impact négatif sur le processus de construction des connaissances, sont identifiées chez certains futurs enseignants. Par ailleurs, l'exploitation des savoirs nécessaires à la caratérisation d'un anion au moyen du nitrate d'argent pour expliquer et interpréter les comportements chimiques des matériaux, montre l'existence de difficultés. En plus, l'utilisation des réactifs généraux et spéciaux tels que le sulfure d'hydrogène et l'hydroxyde de sodium pour observer des réactions caractéristiques des cations, révèlent une insuffisance des savoirs à mobiliser pour identifier le cation zincique au sein du groupe III. L'élaboration des équations-bilans de précipitation et de déshydratation montre des lacunes disciplinaires multiples chez les futurs enseignants. Ces derniers se montrent incapables de présenter des montages expérimentaux au moyen de schémas, malgré l'importance du savoir faire expérimental qui constitue un élément de réussite, aussi bien dans la recherche des ions en analyse qualitative inorganique, que dans l'enseignement et l'apprentissage de la chimie. Les difficultés rencontrées par les futurs enseignants, défavorisant la construction des concepts chez les apprenants, résultent probablement d'une incohérence entre les besoins disciplinaires pour exercer le métier d'enseignant et la formation initiale.

\section{References :}

1. Alexéev, V. (1980). Analyse qualitative. Moscou : Edition Mir.

2. Barlet, R. \& Plouin, D. (1994). L'équation-bilan en chimie un concept intégrateur source de difficultés persistantes. ASTER, 18, 27-56.

3. Bugaje, B.M. (2013). Qualitative chemistry education: the role of the teacher. Journal of Applied Chemistry, 4(5), 10-14. 
4. Calik, M. \& Ayas, A. (2005). A comparison of level of understanding of eighth-grade students and science student teachers related to selected chemistry concepts. Journal of Research in Science Teaching, 42(6), 638-667.

5. Cetin-Dindar, A. \& Geban, O. (2011). Development of a three-tier test to assess high school students'understanding of acids and bases. Procedia Social and Behavioral Sciences, 15, 600-604.

6. Charlot, G. (1974). Chimie analytique quantitative. I Méthodes chimiques et physico-chimique ( $2^{\mathrm{e}}$ éd.). Paris : Masson et Cie.

7. Chevallard, Y. (1985). La transposition didactique: du savoir savant au savoir enseigné. Grenoble : La Pensée sauvage.

8. Chou, C.Y. (2002). Science teachers'understanding of concepts in chemistry. Proceedings of the National Science Council, 12(2), 73-78.

9. Clyde, M. \& Mary, E.C. (1980). Chemistry : Inorganic qualitative analysis in the laboratory. New York : Academic press.

10. Cooley, J.H. \& Williams, R.V. (1999). Qualitative analysis in the beginning organic laboratory. Journal of Chemical Education, 76, 1117-1120.

11. Cormier, C. (2014). Étude des conceptions alternatives et des processus de raisonnement des étudiants de chimie du niveau collégial sur la molécule, la polarité et les phénomènes macroscopiques. Thèse de doctorat. Montréal : Université de Montréal.

12. De Jong, O., Acampo, J. \& Verdonk, A. (1995). Problems in teaching the topic of redox reactions : actions and conceptions of chemistry teachers. Journal of Research in Science Teaching, 32(10), 1097-1110.

13. Dessart, A., Jodogne, J. \& Paul, J. (1973). Chimie analytique. Bruxelles : Edition A, De Boeck.

14. Fresenius, D.C.R. (1845). Précis d'analyse chimique qualitative, ou traité des opérations chimiques, des réactifs de leur action sur les corps les plus répondus. Paris : Portin, Masson et Cie Libraires.

15. Garforth, F.M., Johnsmne, A.H. \& Lazonby, J.N. (1976). Ionic equations: difficulties in understanding and use. Education in Chemistry, 13, 72-75.

16. Givry, D. \& Andreucci, C. (2015). Un schéma vaut-il mieux qu'un long discours ? Éducation et Didactique, 9(1), 119-141.

17. Guy, J.S. (1928). Objectives in the teaching of qualitative analysis. Journal of Chemical Education, 5(5), 573-575.

18. Hamlyn, R., Matthews, P. \& Shanahan, M. (2017). Young people's views on science education: Science education tracker research report. United Kingdom : The royal society.

19. Highton, H.P. (1898). An introduction to practical quantitative analysis. Nature, 59, 172-173. 
20. Imène, M.-K., Dumon, A. \& Ayadi-Trabelsi, M. (2011). Le savoir à enseigner relatif à la transformation chimique en première année de lycée en Tunisie et sa perception par les enseignants. Revista Electrónica de Enseñanza de las Ciencias, 10(1), 153-177.

21. Johnstone, A.H. (1993). The development of chemistry teaching : a changing response to a changing demand. Journal of Chemical Education, 70, 701-705.

22. Johnstone, A.H. \& Wham, A.J.B. (1982). The demands of practical work. Education in Chemistry, 19, 71-73.

23. Köller, H.-G., Olufsen, M., Stojanovska M. \& Petruševski, V. (2015). Practical work in chemistry, its goals and effects. Dans I. Maciejowska \& B. Byers (eds.), A guidebook of good practice for the pre-service training of chemistry teachers (pp.87-106). Krakow : Faculty of Chemistry.

24. Lenton, G. \& Turner, L. (1999). Student-teachers' grasp of science concepts. School Science Review, 81(295), 67-72.

25. Lin, H.S., Cheng, H.J. \& Lawrenz, F. (2000). The assessment of students and teachers understanding of gas laws. Journal of Chemical Education, 77(2), 235-237.

26. Mendham, J., Denney, R.C., Barnes, J.D. \& Thomas, M. (2006). Analyse chimique quantitative de Vogel ( $6^{\mathrm{e}}$ éd.). Bruxelles : De Boeck.

27. MENFP (Ministère de l'éducation nationale et de la formation professionnelle). (2015). Programmes et orientations pédagogiques pour l'enseignement de la physique-chimie en cycle secondaire collégial : Maroc.

28. Nakiboğlu, C. \& Nakiboğlu, N. (2019). Exploring prospective chemistry teachers'perceptions of precipitation, conception of precipitation reactions and visualization of the sub-microscopic level of precipitation reactions. Chemistry Education Research and Practice, 20, 873-889.

29. Nguessan, S.K. (2016). Les futurs enseignants de physique-chimie et le concept d'atome. Quelques représentations, difficultés et obstacles identifies lors de leur formation professionnelle. International Journal of Contemporary Applied Sciences, 3(2), 241- 259.

30. Parmenov, K.I. \& Syroezhkin, I.T. (1963). Practical work in chemistry in the secondary school. Soviet Education, 5(3), 32-36.

31. Quilez-Pardo, J. \& Solaz-Portoles, J.J. (1995). Students and teachers misapplication of Le Chatelier's principle: Implications for the teaching of chemical equilibrium. Journal of Research in Science Teaching, 32(9), 939-957. 
32. Randall, W.H. \& Holly, M.B. (2012). Utilizing problem-based learning in qualitative analysis lab experiments. Journal of Chemical Education, 89, 254-257.

33. Schraw, G. (1998). Promoting general metacognitive awareness. Instructional Science, 26, 113-125.

34. Shamai R. \& Stavy, R. (1986).Teaching an introductory course in qualitative analysis, in order to enhance learning general chemistry. Journal of Chemical Education, 63(8), 707-708.

35. Syed Mustapha, S.M.F.D., Pang, J.S. \& Zain, S.M. (2002). Application qualitative process theory to qualitative simulation and analysis of inorganic chemical reaction. Dans N. Agell \& J.A. Ortega (eds.), $16^{\text {th }}$ international workshop of qualitative reasoning (pp. 177-184). Barcelona: Spain.

36. Tan, D.K.C. (2002). Content framework for teaching and learning inorganic qualitative analysis at the high school level. Australian Journal of Education in Chemistry, 58, 7-12.

37. Tan, K.C.D. (2005). Pre-service teachers' conceptions of basic inorganic qualitative analysis. Canadian Journal of Science, Mathematics and Technology Education, 5(1), (7-20).

38. Tan, D.K.C., Goh, N.-K., Chia, L.-S. \& David, F.T. (2002). Thinking and understanding in qualitative analysis practical work. Teaching and Learning, 23(1), 69-75.

39. Tan, K.C.D., Goh, N.-K., Chia, L.-S., \& Treagust, D.F. (2001). Secondary students perceptions about learning qualitative analysis in inorganic chemistry. Research in Science and Technological Education, 19(2), 223-234.

40. Tan, D.K.C., Goh, N.-K., Chia, L.-S. \& Treagust, D.F. (2004). Qualitative analysis practical work. An instructional package. School Science Review, 85(313), 97-102.

41. Theodore, D.K. (1929). The teaching of qualitative analysis in secondary schools. Journal of Chemical Education, 6(3), 486-492.

42. Valanides, N. (2000). Primary student teachers' understanding of the particulate nature of matter and its transformation during dissolving. Chemistry Education : Research and Practice in Europe, 1(2), 249-262.

43. Večeřa, M. \& Gasparič, J. (1971). Aims and methods of organic qualitative analysis. Dans M. Vecera (ed.), Detection and identification of organic compounds (pp. 15-19). Springer : Boston, MA.

44. Vogel, A.I. (1979). Vogel's textbook of macro and semimicro quantitative inorganic analysis ( $5^{\mathrm{e}}$ éd.). London and New York: Longman. 
45. Wandersee, J. H., Mintzes, J.J. \& Novak, J.D. (1994). Research on alternative conceptions in science. Dans D.L. Gabel (ed.), Handbook of research on science teaching and learning (pp.177-210). New York : Macmillan.

46. Willame, B. \& Snauwaert, P. (2015). Les difficultés rencontrées dans l'apprentissage du concept de concentration en chimie. Spiral-E. Revue de recherches en éducation, supplément électronique, 55(1), 177-205. 